\title{
COMPUTATIONAL STRATEGIES FOR ITERATIVE SOLUTIONS OF LARGE FEM APPLICATIONS EMPLOYING VOXEL DATA
}

\author{
B. VAN RJETBERGEN, H. WEINANS AND R. HUISKES \\ Biomechanics Section, Institute of Orthopaedics, University of Nijmegen, The Netherlands \\ B. J. W. POLMAN \\ Department of Mathematics, University of Nijmegen, The Netherlands
}

\begin{abstract}
SUMMARY
FE-models for structural solid mechanics analyses can be readily generated from computer images via a 'voxel conversion' method, whereby voxels in a two- or three-dimensional computer image are directly translated to elements in a FE-model. The fact that all elements thus generated are the same creates the possibilities for fast solution algorithms that can compensate for a large number of elements. The solving methods described in this paper are based on an iterative solving algorithm in combination with a uniqueelement Element-by-Element (EBE) or with a newly developed Row-by-Row (RBR) matrix-vector multiplication strategy. With these methods it is possible to solve FE-models on the order of 10 3-D brick elements on a workstation and on the order of $10^{6}$ elements on a Cray computer.

The methods are demonstrated for the Boussinesq problem and for FE-models that represent a porous trabecular bone structure. The results show that the RBR method can be 3.2 times faster than the EBE method. It was concluded that the voxel conversion method in combination with these solving methods not only provides a powerful tool to analyse structures that can not be analysed in another way, but also that this approach can be competitive with traditional meshing and solving techniques.
\end{abstract}

KEY WORDS: structural mechanics; voxel conversion; large FEM applications; iterative solving; element-by-element; row-by-row

\section{INTRODUCTION}

In the last decade, FE-methods have developed into the direction of universal tools for all kinds of linear and non-linear structures. Sophisticated element formulations in combination with improved meshing methods, using CAD information that can be directly fed to FE pre-processor codes, have made it possible to mesh every construction that can be manufactured. The challenge in the development of automatic mesh generators has always been to create methods which provide user-friendliness on the one hand, and cost-effective meshes on the other, while maintaining adequate mesh refinement for FE accuracy. This has generally resulted in methods which create non-uniform mesh density, using refinement where it matters, but saving elements where it does not. If however, the number of elements became irrelevant, with, for example, as many elements in a cross section of the FE-model as the number of pixels on a computer monitor, it would be possible to use a regular pattern of equally sized cubic elements, and still describe the geometry of the structure as accurate as it is represented on the monitor. The two- or threedimensional data set displayed on the monitor can then be directly translated to a FE-model by 
converting voxels to equally sized brick elements. Compared to traditional meshing methods, this 'voxel conversion' method is far more simple and faster, providing maximal user friendliness while reducing the matter of accuracy to one single parameter, the (one-dimensional) size of the base elements. The fact that all elements thus generated have the same shape, size and orientation creates the possibilities for fast solution procedures, which can compensate for the large number of elements required in such an approach. In this paper, two of such methods, one based on an element-by-element algorithm, the other on a newly developed 'row-by-row' algorithm, are explained. With these methods, the approach outlined here is indeed possible for data sets on the order of $10^{5}-10^{6}$ voxels.

The new methods discussed here were born out of necessity. In the field of modelling biological structures, problems still arise when constructing even a simple FE-mesh. The irregular shape of biological tissues and their orthotropic material properties make it very difficult to create models that can describe these materials reasonably well. Trabecular bone is such a biological tissue that, due to its load carrying abilities, is of great mechanical interest. It is a porous material with an extremely irregular internal architecture made of 'struts' and 'plates'. This spongy-like internal geometry determines the quality of the bone in terms of mechanical strength and stiffness. A precise description of the architecture and its mechanical consequence is fundamental for the study of the behaviour of bone. Biological processes in bone and bone failure depend on the local mechanical conditions. Hence, in order to study these processes, it is important to evaluate the stresses and strains at the microstructural or tissue level of bone. However, a detailed evaluation of tissue stresses and strains has been inhibited for two reasons. First, the irregularity of the trabecular structures makes it very difficult to create a geometrically accurate FE-model. Second, to obtain both relevant tissue and apparent properties a reasonably large region of trabecular bone must be represented in detail. Such a model must be built of a great number of elements, thus making the solving of the resulting FE-problem very expensive, if possible at all. Recently, methods have been described to generate three-dimensional voxel data sets that can describe the trabecular structure at the microscopic level in detail. ${ }^{1.2}$ Such data sets in combination with the voxel conversion method have been successfully used to overcome the mesh generation problem. ${ }^{3-6}$ However, the large number of elements generated by the voxel conversion method has restricted the application of this method to small pieces of bone (up to $2.3 \mathrm{~mm}$ cubes).

The aim of the present study was to develop numerical methods that fully explore the unique element concept, thus enabling the use of the voxel conversion FE-method to study a piece of trabecular bone that is large enough to allow for proper continuum assumptions. ${ }^{7}$

In the following paragraphs, it is demonstrated first how large such a FE-model is in terms of number of elements and nodes. Based on these two parameters, a fractal dimension is introduced that is very convenient for estimating the accuracy of the FE-mesh and the computational needs for solving the FE-problem. To solve the resulting large scale FE-problems, the Preconditioned Conjugate Gradient method ${ }^{8,9}$ is used. The limiting factor with this procedure is the calculation of the global stiffness matrix-vector product. It is shown first that the actual assembly of the total stiffness matrix is not desirable. Two alternative solution strategies that do not require this assembly are demonstrated. In the first method a unique-element approach of the element by element technique is used. Although this concept was recognized before, ${ }^{10-12}$ the practical applications of this approach were always limited since, as yet, few FE-model are built of unique elements. The second method makes use of a newly developed 'row-by-row' algorithm that allows a very fast assembly of a row of the matrix when needed for the multiplication. The computational requirements and performance of both methods are demonstrated for the well-known Boussinesq problem and for FE-models representing a cube of trabecular bone digitized with different voxel 
resolutions. Computational results are presented for a Cray YMP computer and for a commonly used engineering workstation (Silicon Graphics Iris Indigo).

Although the present study focusses on the 3-D trabecular bone models, the method can be advantageous to study any porous, irregular, or even homogenous structure, for which voxel data can be obtained.

\section{THE VOXEL CONVERSION METHOD}

\section{Construction of digitized meshes of a trabecular bone specimen}

The 3-D Serial Reconstruction technique ${ }^{2}$ was used to determine a detailed description of the trabecular geometry. This method makes use of a microtome and a video camera to obtain images of sequential, thin slices of bone. After digitization of the images, a set of voxel information is created. A voxel in the digitized data set can represent either bone or interstitial fluid. From this data set a FE-model can be created by converting each voxel that represents bone to an equally sized three-dimensional eight-node brick element and assigning global node numbers to the elements (Appendix I). The resolution for digitization should be chosen relative to the size of typical structures. For trabecular bone the typical structures are the trabeculae which are approximately $100 \mu \mathrm{m}$ in thickness. To create a data set that has on average $2 \times 2$ voxels in a cross section of the typical structures, a resolution of at least $50 \mu \mathrm{m}$ is required.

For the present study, a $4.8 \times 4.8 \times 4.8 \mathrm{~mm}$ cube of trabecular bone was digitized in this way. A resolution of $40 \mu \mathrm{m}$ was chosen in each direction, i.e. the total number of voxels in the data set $N_{\text {vox }}$ equalled $120^{3}=1728000$. In this data set 530571 voxels represented bone, thus the volume fraction of the bone equaled $530571 / 120^{3}=0 \cdot 31$. After converting the bone voxels to elements, a FE-model with 530571 elements and 755432 nodes was created (Figure 1).

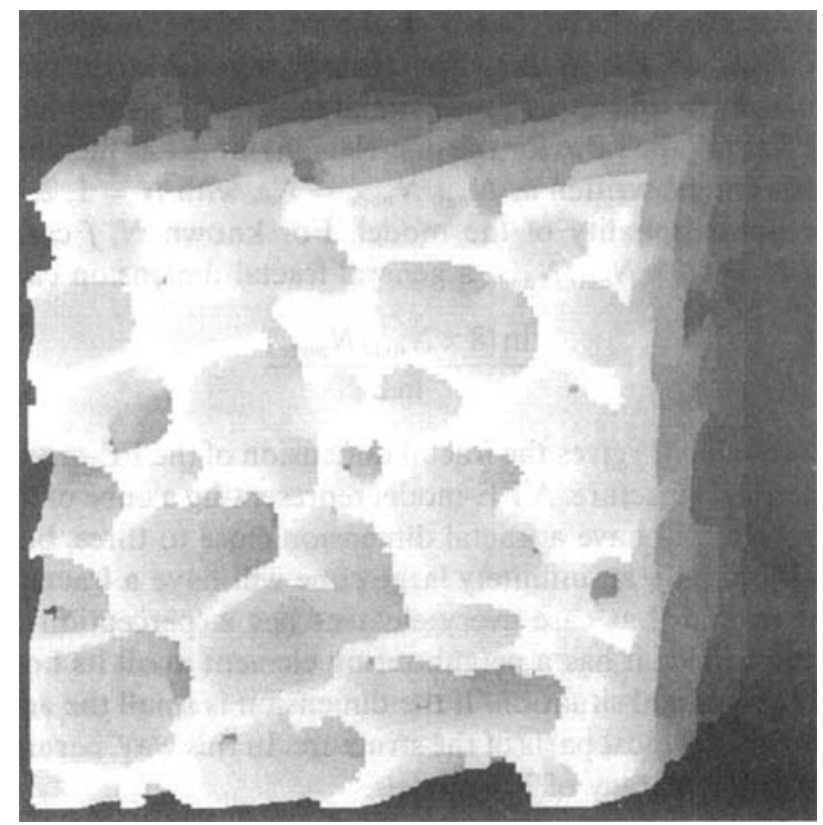

Figure 1. The FE-model of a $4.8 \mathrm{~mm}$ cube of trabecular bone build of 530571 three-dimensional brick elements of $40 \mu \mathrm{m}$ 
Table I. Dimensions of the trabecular bone models

\begin{tabular}{rrrrc}
\hline$N_{\text {vox }}$ & \multicolumn{1}{c}{$N_{\text {nel }}$} & \multicolumn{1}{c}{$N_{\text {node }}$} & $f$ & $\bar{N}_{\text {neighb }}$ \\
\hline $40^{3}$ & 20098 & 42508 & $1 \cdot 92$ & $17 \cdot 6$ \\
$60^{3}$ & 68046 & 121010 & $2 \cdot 17$ & $19 \cdot 8$ \\
$80^{3}$ & 162580 & 259315 & $2 \cdot 33$ & $20 \cdot 3$ \\
$120^{3}$ & 530571 & 755432 & $2 \cdot 49$ & $21 \cdot 7$ \\
\hline
\end{tabular}

The sequential images of the structure were remeshed with resolutions set to 60,80 and $120 \mu \mathrm{m}$. For the $4.8 \mathrm{~mm}$ cube, the number of voxels $N_{\text {vox }}$ in the resulting data sets was $80^{3}, 60^{3}$ and $40^{3}$, respectively. The number of elements and nodes after conversion to FE-models are listed in Table I.

Introduction of a fractal dimension to estimate the accuracy and numerical requirements of digitized meshes

Hughes et al. ${ }^{12}$ have introduced a new concept for the dimension of a mesh build of three-dimensional continuum elements, such that a long strip model is labelled as one-dimensional, a large plate model as two dimensional and a solid cube model as three dimensional.

Depending on its purpose, there are a number of ways to define such a fractal dimension. In their study, Hughes et al. based the fractal dimension on the profile storage to hold the global stiffness matrix, such to differentiate when the EBE strategy is advantageous over a direct method.

In the context of the algorithms proposed in this manuscript, this definition is not so relevant. Therefore, an alternative formulation for the fractal dimension of FE-models made of eight-node brick continuum elements is introduced, based on the ratio of the number of elements $N_{\text {nel }}$ over the number of nodes $N_{\text {node }}$ in the model. For typical, infinite large, models that are to be classified as a zero-, one-, two- and three-dimensional models, this ratio is $1 / 8,1 / 4,1 / 2$ and $1 / 1$, respectively (Figure 2). Taking the ratio for the one-element model as the unit of measure $\varepsilon=1 / 8$, the ratio in these models can be written as $N_{\text {nel }} / N_{\text {node }}=N \varepsilon$, with $N=1,2,4$, and 8 respectively, or $N=2^{f}$, with $f$ the dimensionality of the model. For known $N, f$ can be calculated from $f=\ln (N) / \ln (2)$. Using $N=1 / \varepsilon \times N_{\text {nel }} / N_{\text {node }}$ a general fractal dimension can be defined as:

$$
f=\frac{\ln \left(8 \times N_{\text {nel }} / N_{\text {node }}\right)}{\ln 2}
$$

It should be noted that relation (1) gives the fractal dimension of the FE-model and not the fractal dimension of the geometrical structure. A FE-model representing a cube of limited size, i.e. a fully three-dimensional structure, will have a fractal dimension close to three, but not equal to three. With the present definition, only an infinitely large cube will have a fractal dimension of three, which basically means that in that case every element has a 'perception' of being in a threedimensional configuration, since it has a neighbouring element at all its borders. This is for the accuracy of the model the optimal situation. If the dimension is small the accuracy is poor, since there are only few elements in typical parts of the structure. In this way, parameter $f$ can be used to estimate the resolution and accuracy of FE-models.

For the microstructural FE-models representing trabecular bone, a fractal dimension close to one indicates that the trabeculae are represented by strip-like structures with only one element in 


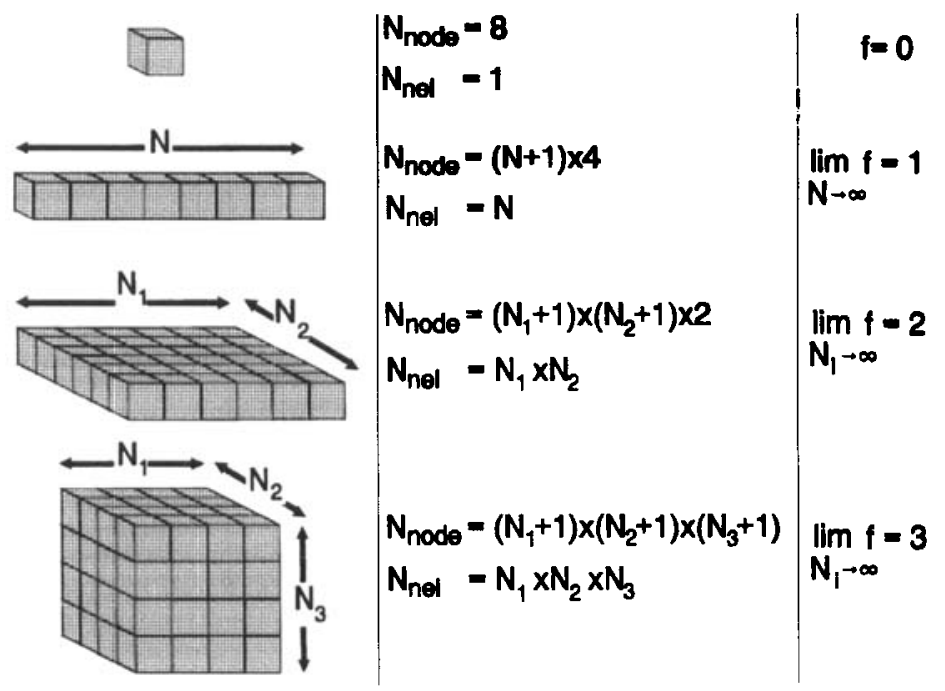

Figure 2. The fractal dimension calculated from equation (1) for four reference models

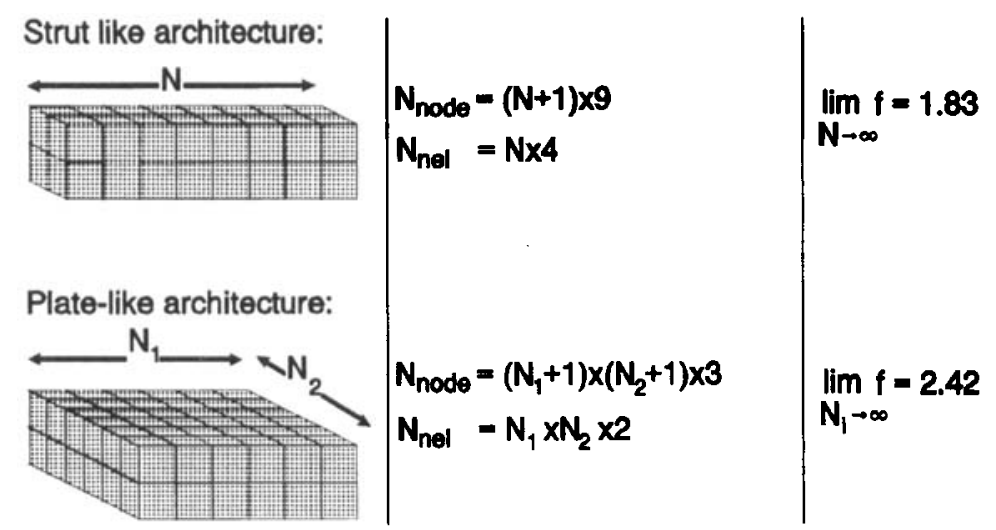

Figure 3. The fractal dimension for a strut-like structure with 4 elements in each cross-section, and for a plate-like structure with 2 element layers

a cross section. It is clear that such FE-models can not represent the geometry of the trabeculae very well and produce poor results when loaded in bending. FE-models with a fractal dimension close to three give a very accurate representation of the trabecular structure, and accurate numerical results. However, such a model will have a large number of elements in the cross section of each trabecula, resulting in time consuming if not unsolvable FE-problems. It was stated before that an acceptable FE-model of a trabecular structure must have at least $2 \times 2$ elements in a trabecular cross-section. For trabecular structures with a strut-like architecture, this criterion can now be translated to the requirement that the fractal dimension of the FE-models must be greater than 1.83 (Figure 3). For constructions with a plate-like architecture a minimum fractal dimension of 2.42 can be calculated (Figure 3 ). 


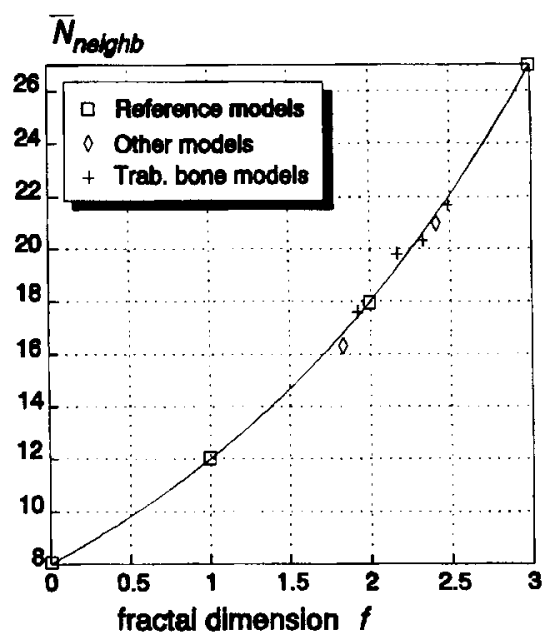

Figure 4. Relation between the fractal dimension and the average number of neighbor nodes for the reference models of Figures 2 and 3 and for the trabecular FE-models

The fractal dimension of the FE-model can also be used to estimate the average number of neighbour nodes $\bar{N}_{\text {neighb }}$ for the nodes in the FE-model. The average number of neighbour nodes multiplied with the number of degrees of freedom per node $N_{\text {dof }}$ is an indication for the number of non-zero entries in a row of the global stiffness matrix for the FE-problem. For the models of Figure 2 the average number of neighbour nodes can easily be calculated. The minimum number is found for the FE-model with a fractal dimension of zero where each node has eight neighbour nodes (including itself) at the corners of the element. For the model with a fractal dimension of one each node has 12 neighbour nodes, for a fractal dimension of two there are 18 neighbour nodes, and in the maximum case, for a model with a fractal dimension of three, each node has 27 neighbour nodes. Fitting a cubic function through these four data points gives the diagram of Figure 4. For other FE-models, the relation between fractal dimension and the average number of neighbour nodes can deviate somewhat from this line. To check for this, the average number of neighbour nodes is explicitly calculated for the FE-models of Figure 3 and for the trabecular models (Table I), and these data points are included in Figure 4. For the piece of trabecular bone with a plate-like architecture (Figure 1), it was calculated that the fractal dimension should be greater than 2.42 . The fractal dimensions for the trabecular bone models with different resolution are specified in Table I. It can be seen that this criterion is met only for the FE-model generated from $40 \mu \mathrm{m}$ voxels $\left(N_{\mathrm{vox}}=120^{3}\right)$. Therefore, this model is considered as typical for a microstructural FE-model of trabecular bone. In the following, computational requirements are demonstrated for this model.

\section{THE FE-APPROACH, A STORAGE AND OPERATION COUNT}

The preconditioned conjugate gradient method to solve the FE-problems

The displacement formulation of the FE-method results in the following set of linear equations:

$$
\mathbf{K u}=\mathbf{f}
$$




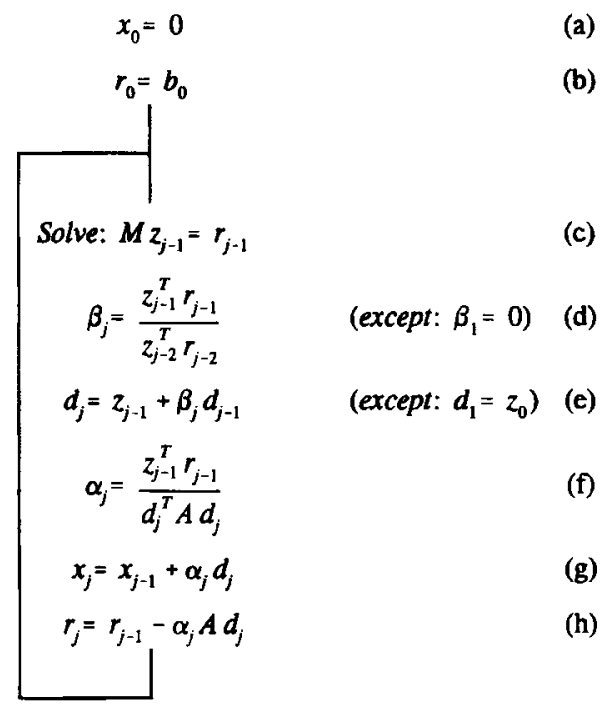

Figure 5. The Preconditioned Conjugate Gradient scheme (taken from Reference 9)

with $\mathbf{K}$ the global stiffness matrix, $\mathbf{u}$ the unknown displacement vector, and $\mathbf{f}$ the external force vector. The order $N_{\text {eq }}$ of the problem equals

$$
N_{\text {eq }}=N_{\text {dof }} \times N_{\text {node }}
$$

with $N_{\text {node }}$ the number of nodes in the mesh and $N_{\text {dof }}$ the number of degrees of freedom for each node. In the 3-D situation there are three degrees of freedom for each nodal point: $N_{\text {dof }}=3$. Matrix K defines the stiffness relations between the degrees of freedom; it is positive definite, symmetric, and very sparse. The bandwidth of $\mathbf{K}$ is determined by the maximum difference in the node numbers of an element multiplied with the number of degrees of freedom at each node. For a FE-model created from a cubic voxel data set with $N$ voxels on each side of the cube, the bandwidth of matrix $\mathbf{K}$ is in the order $N_{\text {dof }} \times N \times N$. For large models with $N=120$ or more, this bandwidth in combination with the order of the matrix inhibits the use of direct solving methods that require filling the band of $\mathbf{K}$. Instead, an iterative solving method can be used. The Preconditioned Conjugate Gradient (PCG) method has been described as an effective method to solve equation (2). ${ }^{8,13,12,9}$ A scheme of the PCG-method as used in the present code, taken from Strang, ${ }^{9}$ is shown in Figure 5. Diagonal scaling was used as a preconditioner. For this, the preconditioning matrix $\mathbf{M}$ is replaced by the diagonal a of the global stiffness matrix $\mathbf{K}$. Solving the set of equations at (c) in the scheme can then be done by multiplying the right-hand side vector by the inverse of a. The total storage requirements for the PCG solving method include the storage of matrix $\mathrm{K}$ and five vectors of order $N_{\mathrm{eq}}$ that are needed during the PCG solving process: the estimated displacement vector $\mathbf{x}$, a residue vector $\mathbf{r}$, a pseudo-residue vector $\mathbf{z}$, a direction vector $\mathbf{d}$, and the diagonal a of matrix $\mathbf{K}$. The right-hand-side of equation (2) contains relatively few non-zero entries and can be stored in compact form by storing only the position and value of the non-zero components. The global stiffness matrix is needed only once per iteration for the matrix-vector multiplication at step (f) in the scheme. The result is stored in vector $\mathbf{z}$, to be used again in step (h). It is shown in the following paragraph that the explicit assembly and storage of $\mathbf{K}$ is not desirable for large problems, not even when using a sparse matrix storage scheme. Two 
alternative algorithms are demonstrated, one based on element-by-element techniques, the other on a newly developed row-by-row technique. Both methods enable the matrix-vector multiplication without storing the global stiffness matrix. As the matrix vector product calculation involves the biggest part of all multiplications needed in each iterative increment, the actual number of multiplications for this operation largely determines the incremental cpu-time. Therefore, the number of multiplications is calculated explicitly for the assembly method and for the two alternative methods.

\section{Storage and operation count for the assembly method}

As matrix $\mathbf{K}$ is very sparse, it is favourable to use a compact storage technique that only stores the non-zero matrix entries and the location of these. ${ }^{14,15}$ The number of non-zero matrix entries in a row of the matrix can be determined from the number of neighbour nodes for the node associated with that row. At row $i$ of matrix $\mathbf{K}$ there are $N_{\text {dof }} \times N_{\text {neighb, n }}$ matrix entries, with $N_{\text {neighb, n }}$ the number of neighbours of node $n$ that corresponds to row $i$. The total number of matrix entries in K can be estimated from $N_{\text {dor }} \times \bar{N}_{\text {neighb }} \times N_{\text {eq }}$, with $\bar{N}_{\text {neighb }}$ the average number of neighbour nodes, which can be estimated from the fractal dimension of the FE-model, as shown in Figure 4. As the matrix is symmetric, $N_{\text {dof }} \times \bar{N}_{\text {neighb }} \times N_{\text {eq }} / 2$ real numbers must be stored to hold matrix K. Another $\left(N_{\text {dof }} \times \bar{N}_{\text {rieighb }}+1\right) \times N_{\text {eq }} / 2$ integers must be stored to reconstruct the location of each term in the matrix. ${ }^{14,15}$ Using equation (3) the total memory requirements to store matrix $K$ and the five iteration vectors in the 3-D situation $\left(M_{\text {ENS }}\right)$ can be expressed as a function of the number of nodes in the FE-model:

$$
\mathrm{MEM}_{\text {Ass }}=\left[9 \times \bar{N}_{\text {neighb }} \times N_{\text {node }} / 2+15 \times N_{\text {node }}\right] \text { words of real }
$$

and

$$
\left[\left(3 \times \bar{N}_{\text {neighb }}+1\right) \times 3 \times N_{\text {node }} / 2\right] \text { words of integer }
$$

For the matrix-vector product calculation, each matrix component is to be multiplied with the corresponding vector component. The total number of multiplications $\left(\mathrm{MUL}_{\mathrm{Ass}}\right)$ thus equals the number of matrix entries:

$$
\mathrm{MUL}_{\text {ASS }}=9 \times \bar{N}_{\text {neighb }} \times N_{\text {node }}
$$

For the typical trabecular bone model with a fractal dimension of 2.49 , the average number of neighbour nodes was $\bar{N}_{\text {neighb }}=21.7$ and the number of nodes $N_{\text {node }}=755432$. From equation (4) it can be calculated that for solving this FE-problem when storing the assembled matrix $\mathbf{K}$, one needs to store 85 Mword of reals and another 75 Mword of integers. Presently, only large supercomputers are equipped with enough core memory to hold this.

The unique-element implementation of the element by element matrix-vector algorithm

With the EBE method, the global stiffness matrix $\mathbf{K}$ is not assembled, but instead, the vectors are disassembled for each element. ${ }^{10,13,12}$ The matrix-vector multiplication then takes place at the element level by multiplying the element stiffness matrix with the corresponding disassembled vectors. For FE-models created from voxel data, this approach is very favourable because, as all elements are identical in size and have the same orientation and material properties, all element stiffness matrices are the same. Thus, only one element stiffness matrix has to be stored (300 words). This reduction even applies when each element would have a different Young's modulus, 


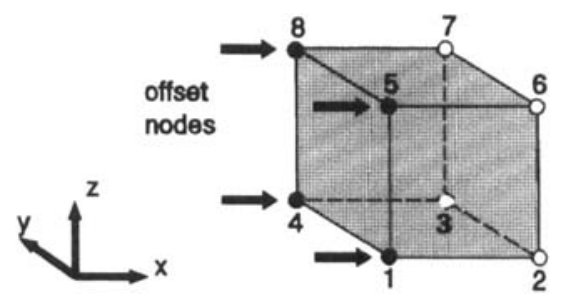

Figure 6. Definition of the four offset nodes at the $x=x_{\min }$ element face, used to define the element connectivity for the EBE method

because the isotropic element stiffness matrix is a linear function of this modulus. For the assembly and disassembly of the vectors, an element localization matrix must be stored, which defines the mapping of the 24 degrees of freedom for each element to the global degree of freedom numbers. As the local and global degree of freedom numbers can be derived from the node numbers, it is sufficient to store an array that defines the mapping of the eight element node numbers to the global node numbers. By using a consequent numbering scheme in the construction, with node numbers increasing with increasing $x$-co-ordinate, the storage requirements can be further reduced by storing only the 'offset node numbers' at the $x=x_{\min }$ face of each element (Figure 6). Thus, for the construction of the element localization matrix $4 \times N_{\text {nel }}$ words of integer have to be stored. Including the five iteration vectors for the Preconditioned Conjugate Gradient method, the total storage requirements for 3-D problems can be estimated from:

$$
\mathrm{MEM}_{\mathrm{EBE}}=\left[15 \times N_{\text {node }}\right] \text { words of real and }\left[4 \times N_{\mathrm{nel}}\right] \text { words of integer }
$$

The reduction in memory requirements compared to the assembly method can be demonstrated for the typical FE-model where, instead of $85 \mathrm{Mword}$ real and 75 Mword integer storage, for the EBE method only $11 \mathrm{Mword}$ real and 2.1 Mword integer storage is needed. For computers that use 8 byte storage for (double) real variables and 4 byte storage for integer variables, the memory requirements expressed in bytes is

$$
\mathrm{MEM}_{\mathrm{EBE}}=120 \times N_{\text {node }}+16 \times N_{\text {nel }} \quad \text { (byte) }
$$

The Cray computer uses 8 byte storage for both real and integer variables. For this computer

$$
\left(\mathrm{MEM}_{\mathrm{EBE}}\right)_{\mathrm{Cray}}=120 \times N_{\text {node }}+32 \times N_{\text {nel }} \text { (byte) }
$$

The number of multiplications for the EBE matrix-vector calculation is determined by the number of elements only. For each 3-D eight-node brick element, the 24 rows of the element stiffness matrix are to be multiplied with the 24 components of the disassembled vector. The total number of multiplications is thus

$$
\mathrm{MUL}_{\mathrm{EBE}}=24 \times 24 \times N_{\text {nel }}
$$

In general, the number of multiplications for the EBE method is larger than for the assembly method. The actual increase in the number of multiplications compared to the assembly method is a function of the number of elements to the number of nodes ratio, and thus of the fractal dimension of the FE-model. For models with a fractal dimension close to three, the number of elements approaches the number of nodes and the average number of neighbour nodes approaches $\bar{N}_{\text {neighb }}=27$, the number of multiplications for the assembly method is then by good 


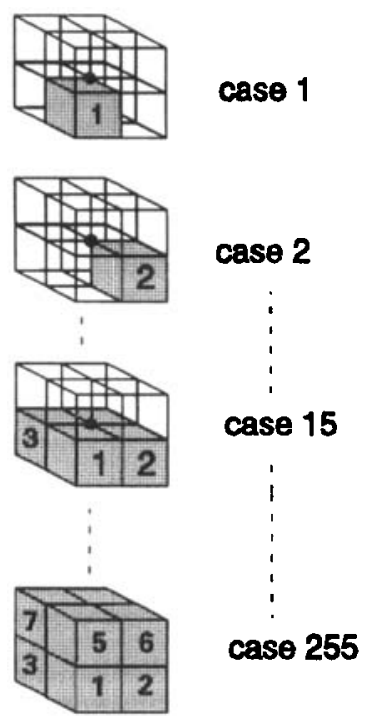

Figure 7. Four of the 255 possible cases

approximation (equation (5)) $\mathrm{MUL}_{\mathrm{Ass}}=9 \times 27 \times N_{\text {nel }}$, and for the EBE method $\mathrm{MUL}_{\mathrm{EBE}}$ $=24 \times 24 \times N_{\text {nel }}$. The increase in multiplications for the EBE method over the assembly method, represented by the ratio $M U L_{E B E}$ over $M U L_{A s s}$, is then 2.37. For models with a fractal dimension less than three, this ratio is less: 1.77 for models with a fractal dimension of two, and 1.33 for a fractal dimension of one.

The row by row matrix-vector algorithm

Another alternative for the full assembly and storage of matrix $\mathbf{K}$ is the assembly and storage of entries in $\mathbf{K}$ only when needed for the matrix-vector multiplication. The row-by-row algorithm is an algorithm in this category that assembles a row of matrix $\mathbf{K}$ every time when it is needed for the multiplication. The row assembly can be done very efficiently by using the fact that only a limited number of row configurations are possible in the global matrix $\mathbf{K}$. These row configurations can be determined on beforehand by analysing all possible neighbour-node configurations for a node in the model. In the voxel-grid situation, each node is surrounded by eight voxels that represent either bone or void space. Thus, after conversion of the bone voxels to elements in the FE-model, there are $2^{8}=256$ possible environments for a node in the mesh. The case in which there is no element at all can be excluded, leaving 255 cases of interest (Figure 7). Each case can be uniquely defined from the numbering scheme as shown in Table II. The case number of each node can be determined during the preprocessing stage by checking for neighbouring elements (Appendix II). Each case results in a specific set of matrix entries at the $N_{\text {dof }}$ matrix rows that correspond to the node. The configuration of these rows can be pre-assembled for each nodal case. By creating a subroutine library that contains the pre-assembled cases, the matrix entries in a row of the matrix can be found easily if the 'case number' of the node is known. To assemble a row of the global stiffness matrix, the location of each term must be determined. For this a nodal localization matrix is used which defines the mapping of the degrees of freedom numbers of the base cube of Figure 8 to the global degree of freedom numbers. As the degree of freedom numbers can be 
Table II. Numbering scheme for the 255 cases

\begin{tabular}{|c|c|c|c|c|c|c|c|c|}
\hline \multirow[b]{2}{*}{ Case number } & \multicolumn{8}{|c|}{ Element } \\
\hline & 1 & 2 & 3 & 4 & 5 & 6 & 7 & 8 \\
\hline 1 & $\mathrm{x}$ & 0 & 0 & 0 & 0 & 0 & 0 & 0 \\
\hline 2 & 0 & $\mathrm{x}$ & 0 & 0 & 0 & 0 & 0 & 0 \\
\hline 3 & $\mathbf{x}$ & $x$ & 0 & 0 & 0 & 0 & 0 & 0 \\
\hline \multirow{2}{*}{\multicolumn{9}{|c|}{. }} \\
\hline & & & & & & & & \\
\hline 255 & $\mathbf{x}$ & $\mathrm{x}$ & $\mathbf{x}$ & $\mathrm{x}$ & $\mathrm{x}$ & $\mathrm{x}$ & $\mathrm{x}$ & $\mathrm{x}$ \\
\hline
\end{tabular}

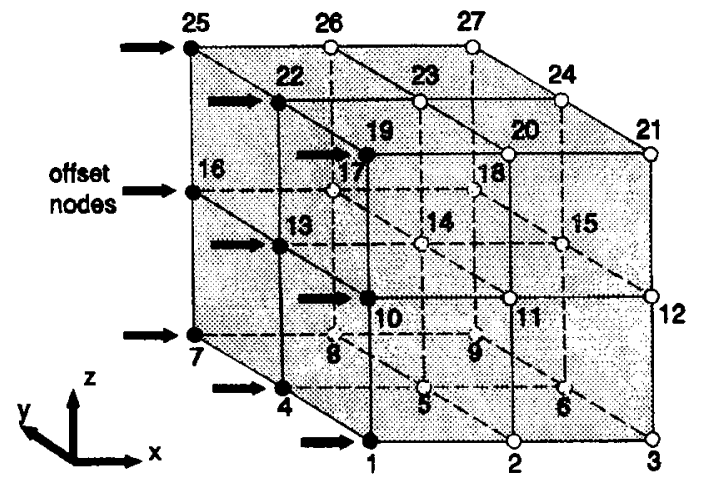

Figure 8. Definition of the offset nodes at the $x=x_{\min }$ face of the basic cube used to define the nodal connectivity for the RBR method

derived from the node numbers, it is sufficient to store an array that defines the mapping of the 26 node numbers surrounding the central node of the base-cube of Figure 8 relative to the global node numbers. This requires the storage of 26 integers per node number. Using the consequent numbering scheme mentioned before, with node numbers increasing with increasing $x$-co-ordinate, the storage requirements can be further reduced by storing only the global node numbers of nine 'offset node numbers' at the $x=x_{\min }$ face of the base-cube of Figure 8 for each nodal point. The node numbers of the midnodes of the cube and of the nodes at the plane $x=x_{\max }$ can be derived from the offset numbers by adding one or two, respectively, to the corresponding offset number. Using this numbering scheme, $N_{\text {node }} \times 9$ integer words are needed to store the nodal offset numbers (Appendices III and IV).

The steps involved for using the RBR matrix-vector multiplication are summarized in Figure 9. Including the five iteration vectors the total storage requirements for this method in the 3-D situation can be written as a function of the number of nodes:

$$
\operatorname{MEM}_{\mathrm{RBR}}=\left[15 \times N_{\text {node }}\right] \text { words of real and }\left[9 \times N_{\text {node }}\right] \text { words of integer }
$$




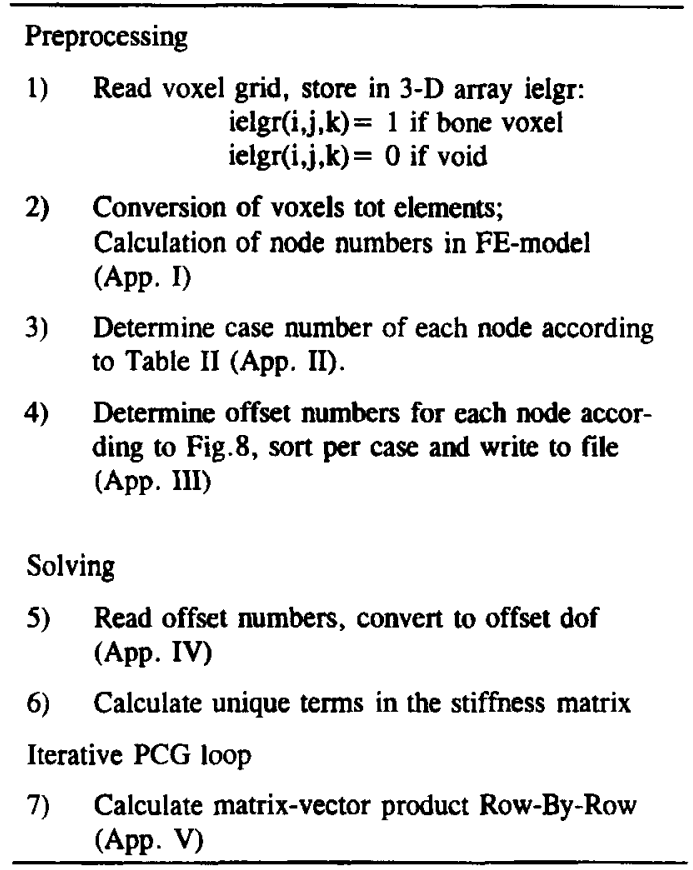

Figure 9. Overview of the steps involved with the RBR solving strategy

The memory requirements in bytes for computers that use 8 byte storage for (double) real variables and 4 byte storage for integer variables can be determined as

$$
\mathrm{MEM}_{\mathrm{RBR}}=156 \times N_{\text {node }} \quad(\text { byte })
$$

and for the Cray computer with 8 byte storage for both real and integer variables:

$$
\left(\mathrm{MEM}_{\mathrm{RBR}}\right)_{\mathrm{Cray}}=192 \times N_{\text {node }} \quad(\text { byte })
$$

An advantage of the row-by-row method is the reduced number of multiplications compared to the assembly method, due to symmetries in the case configurations. Assembly methods require the multiplication of all matrix entries that are assembled in a row of the matrix. For the RBR method however, the zero entries due to symmetries can be removed from the case libraries, thereby saving the cost of multiplying with zero entries during the matrix-vector multiplication. The number of symmetries $N_{\text {sym }}$ for the three matrix rows that correspond to a node in the construction can be determined from its case number, as each case has its specific number of symmetries. As the distribution of the case numbers is a function of the fractal dimension, the number of symmetries can also be expressed as a function of the fractal dimension. The graph of Figure 10 shows the average number of symmetries per node for the reference models of Figures 2 and 3 as a function of their fractal dimension. It can be seen from this graph that the reduction in multiplications due to symmetries is a function of the fractal dimension. For the trabecular bone models the actual number of symmetries was explicitly calculated from the case-number distribution; these data points are also included in Figure 10. The average number of 


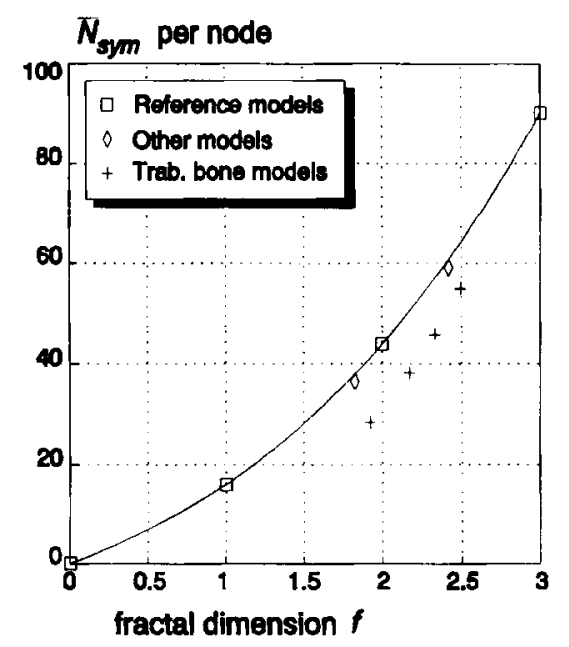

Figure 10. The average number of symmetries $\bar{N}_{\mathrm{sym}}$ as a function of the fractal dimension of the FE-model

symmetries per node for a trabecular bone model is less than that for a reference model with the same fractal dimension, because of the more 'jagged' boundaries in the trabecular models. Hence, the values found for the reference models represent the upper boundaries for the number of symmetries.

The actual number of multiplications for the RBR matrix-vector calculation can now be determined from the relation:

$$
\mathrm{MUL}_{\mathrm{RBR}}=\left(9 \times \bar{N}_{\text {neighb }}-\bar{N}_{\text {sym }}\right) \times N_{\text {node }}
$$

For models with a fractal dimension of three, assembly methods require the multiplication of $9 \times \bar{N}_{\text {neighb }}=243$ matrix entries for each node in the FE-model, of which $\bar{N}_{\text {sym }}=90$ zero entries. Thus, using the RBR method, the number of multiplications can be reduced by 37 per cent compared to the assembly method. For FE-models with a fractal dimension of two, 44 of the 162 matrix entries are zero, reducing the cpu-time by 27 per cent and for FE-models with a fractal dimension of one a 15 per cent reduction is obtained.

For the RBR-method, the actual matrix-vector product calculation is reduced to a nodal-casedependent subroutine call (Appendix IV). To avoid a case-subroutine call for each node, all nodes of the same case are grouped and processed together in the corresponding subroutine. The multiplications in each case subroutine can be performed in a vectorized manner, with a vector length that equals the number of nodes for that case.

\section{Test problems for a performance study of the EBE and RBR solution method}

The EBE and the RBR method are demonstrated for two groups of problems. The first group represents a set of three dimensional Boussinesq problems; ${ }^{12,13}$ a half-space with a unit point load. The number of elements in the Boussinesq cube was chosen equal to the number of voxels in the trabecular bone models: $40^{3}, 60^{3}, 80^{3}$, and $120^{3}$, resulting in FE-models with 64000 to 1728000 elements (Table III). The second group consists of the four trabecular bone models digitized with different resolutions. For these models a uniform unit displacement in the 
Table III. Dimensions of the Boussinesq problems

\begin{tabular}{rrrr}
\hline$N_{\text {vax }}$ & \multicolumn{1}{c}{$N_{\text {nel }}$} & \multicolumn{1}{c}{$N_{\text {node }}$} & $f$ \\
\hline $40^{3}$ & 64000 & 68921 & 2.89 \\
$60^{3}$ & 288000 & 226981 & 2.93 \\
$80^{3}$ & 512000 & 531441 & 2.95 \\
$120^{3}$ & 1728000 & 1771561 & 2.96 \\
\hline
\end{tabular}

$z$-direction was applied to the top face of the cube. At the bottom face the displacements in the $z$-direction were constrained. All other faces of the cube were unconstrained. In all models the material properties were linear elastic and isotropic with a Young's modulus of $1000 \mathrm{MPa}$ and a Poisson's ratio of $0 \cdot 3$.

The iterative process was terminated as soon as the norm of the residual vector over the norm of the reaction force vector was less than $1 \times 10^{-4}$ :

$$
\frac{\left|F_{\text {res }}\right|}{\left|F_{\text {reac }}\right|} \leqslant 1 \times 10^{-4}
$$

For the Boussinesq problems, the norm of the reaction force equals the norm of the externally applied load: $\left|F_{\text {reac }}\right|=1 \cdot 0$. For the trabecular bone models the reaction force vector was calculated at increment $1,2,4,8,16, \ldots$ The norm of the last update of the reaction force vector was used to test criterion (14) at the next increments. All problems were solved using one processor of a Cray YMP/4 computer with $64 \mathrm{Mword}$ of memory. To demonstrate the performance of both methods on other computers, the problems also ran on an engineering Risc workstation (Silicon Graphics Iris Indigo with Mips R4000 processor, 48 Mbyte core memory and 1 Mbyte cache). All problems ran 'in-core'. The number of iterations and the total cpu-time on each platform was recorded.

\section{NUMERICAL RESULTS FOR THE EBE AND THE RBR SOLUTION METHOD}

\section{Storage requirements}

The storage requirements for both the EBE and for the RBR method (equations (6) and (10)) can be expressed as a function of the number of elements only, if the fractal dimension of the FE-model is known. For a FE-model with a fractal dimension close to three (such as the Boussinesq problem), it can be calculated from equations (1), (8), and (12) that the RBR method needs 26.3 per cent more workspace than the EBE-method on the Cray computer. On the workstation, 14.7 per cent more workspace is needed. For a fractal dimension close to two (such as the trabecular models), 41 per cent more workspace is needed for the RBR method compared to the EBE method on the Cray, and 22 per cent more on the workstation. The relation between the number of elements and the core memory needed on the Cray computer for the EBE and for the RBR methods is shown by three lines in Figure 11 for models with a fractal dimension of one, two and three.

The actual storage requirements as listed in Tables IV and V include the storage of additional problem-size dependent, but relatively small vectors for prescribed and suppressed displacements and for the prescribed load. It does not include the storage of the code itself (approximately 
Table IV. Memory requirements for the 3-D Boussinesq problems

\begin{tabular}{crrrcc}
\hline & \multicolumn{2}{c}{ Cray YMP } & & \multicolumn{2}{c}{ Risc Workstation } \\
\cline { 2 - 3 } \cline { 5 - 6 }$N_{\text {vox }}$ & $\begin{array}{c}\text { EBE } \\
\text { (Mbyte) }\end{array}$ & $\begin{array}{c}\text { RBR } \\
\text { (Mbyte) }\end{array}$ & & $\begin{array}{c}\text { EBE } \\
\text { (Mbyte) }\end{array}$ & $\begin{array}{c}\text { RBR } \\
\text { (Mbyte) }\end{array}$ \\
\hline $40^{3}$ & $10 \cdot 36$ & $13 \cdot 27$ & & $9 \cdot 32$ & $10 \cdot 77$ \\
$60^{3}$ & 34.24 & $43 \cdot 67$ & & 30.74 & $35 \cdot 45$ \\
$80^{3}$ & $\mathbf{8 0 . 3 2}$ & $102 \cdot 20$ & & - & - \\
$120^{3}$ & $268 \cdot 24$ & $340 \cdot 49$ & & - & - \\
\hline
\end{tabular}

Table V. Memory requirements for the trabecular bone models

\begin{tabular}{rrrrrr}
\hline & \multicolumn{2}{c}{ Cray YMP } & \multicolumn{2}{c}{ Risc workstation } \\
\cline { 2 - 3 } \cline { 5 - 6 }$N_{\text {vox }}$ & $\begin{array}{c}\text { EBE } \\
\text { (Mbyte) }\end{array}$ & $\begin{array}{c}\text { RBR } \\
\text { (Mbyte) }\end{array}$ & \multicolumn{2}{c}{$\begin{array}{c}\text { EBE } \\
\text { (Mbyte) }\end{array}$} & $\begin{array}{c}\text { RBR } \\
\text { (Mbyte) }\end{array}$ \\
\hline $40^{3}$ & $5 \cdot 77$ & $8 \cdot 18$ & 5.44 & 6.64 \\
$60^{3}$ & $16 \cdot 74$ & $23 \cdot 27$ & 15.64 & 18.90 \\
$80^{3}$ & $36 \cdot 39$ & $49 \cdot 85$ & $33 \cdot 76$ & $40 \cdot 50$ \\
$120^{3}$ & $107 \cdot 76$ & $145 \cdot 17$ & - & - \\
\hline
\end{tabular}

1 Mbyte for the EBE code and 2 Mbyte for the RBR code). The data points of Tables IV and $\mathrm{V}$ are also included in Figure 11.

\section{Computational time}

The number of multiplications for the different matrix-vector product calculation (equations (5), (9) and (13)) can also be expressed as a function of the number of elements in the FE-model, if the fractal dimension of the model is known. The number of multiplications per element for the reference models when using the EBE, the assembly and the RBR method is shown in Figure 12 as a function of the fractal dimension. For a FE-model with a fractal dimension close to three (such as the Boussinesq problem), it follows that the EBE method needs 3.76 times more multiplications than the RBR method. For models with a fractal dimension close to two, 2.43 times more multiplications are needed. The number of multiplications per element was explicitly calculated for the trabecular models, these data points are included in the graph of Figure 12.

From the actual cpu-time per iteration for the Boussinesq problems shown in Table VI it can be seen that the speed increase for the RBR over the EBE method on the Cray computer is a little bit less than calculated from equations (9) and (13): a factor of 3.0 for the model with $N_{\text {vox }}=40^{3}$ to 3.22 for the model with $N_{\text {vox }}=120^{3}$. This is due to the additional gather and scatter operations and vector-vector multiplications in each iterative increment which are not included in equations (9) and (13). For the trabecular bone models, speed-up factors of 1.85 for the model with $N_{\text {vox }}=40^{3}$ to 2.37 for the model with $N_{\text {vox }}=120^{3}$ were found (Table VII). The speed-up factor on the workstation is somewhat less: approximately 2.5 for the Boussinesq problems to 1.5 for the 

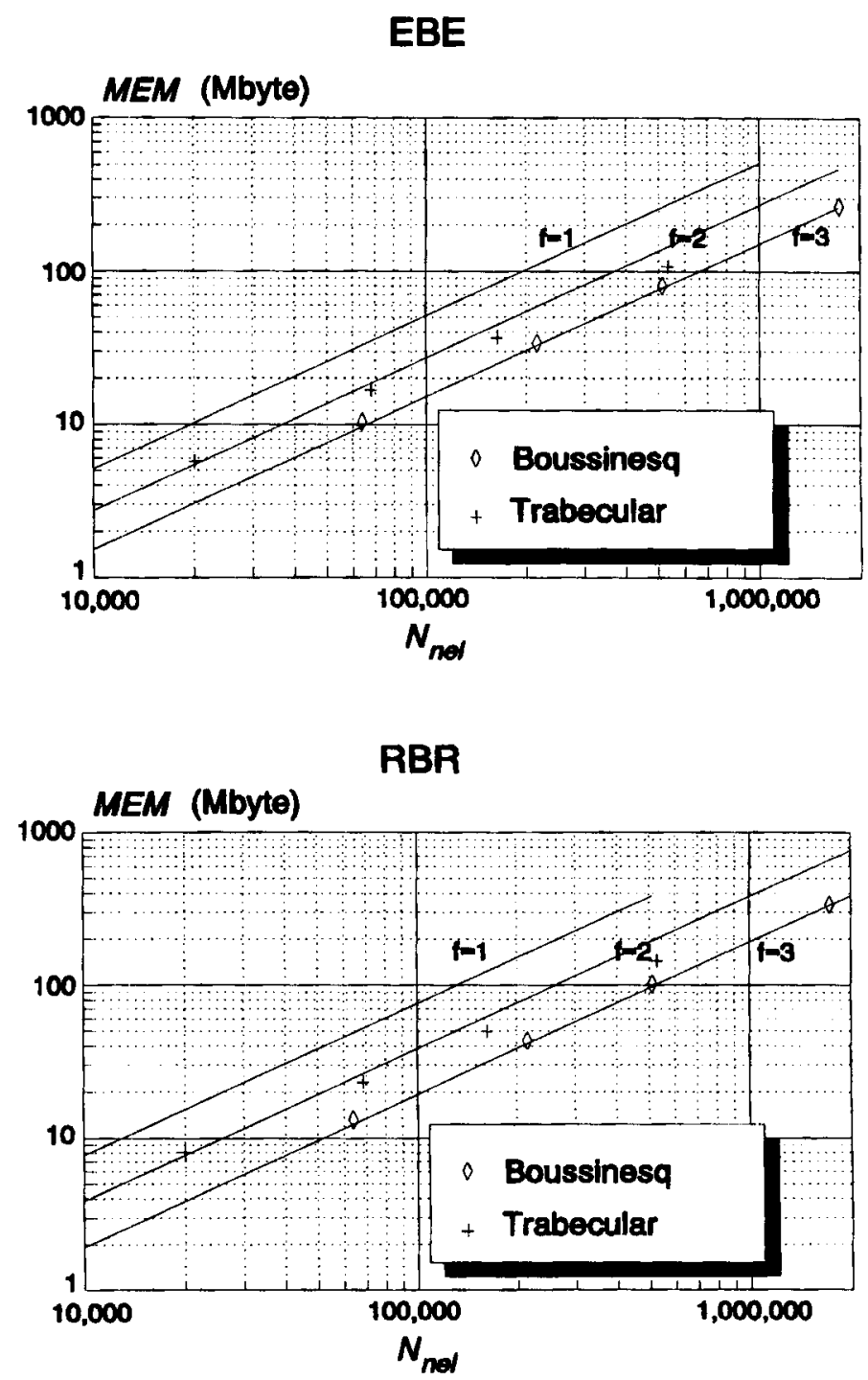

Figure 11. Total core memory needed on a Cray computer to solve the Boussinesq problems ( $\diamond)$ and the trabecular problems (+). Results for the EBE method are shown on top, for the RBR method on the bottom

trabecular models. This indicates that the cache-hit rate for the RBR-algorithm is less than for the EBE algorithm. The total cpu-time to solve the trabecular bone models on both the Cray and the workstation is shown in Figure 13. It can be seen that the largest model can be solved in approximately $1 \mathrm{~h}$ on the Cray computer when using the RBR algorithm.

\section{DISCUSSION}

The present study has demonstrated that it is possible to solve 3-D FE-models with in the order of $10^{5}-10^{6}$ unique elements. Such solving methods in combination with the 'voxel conversion' 


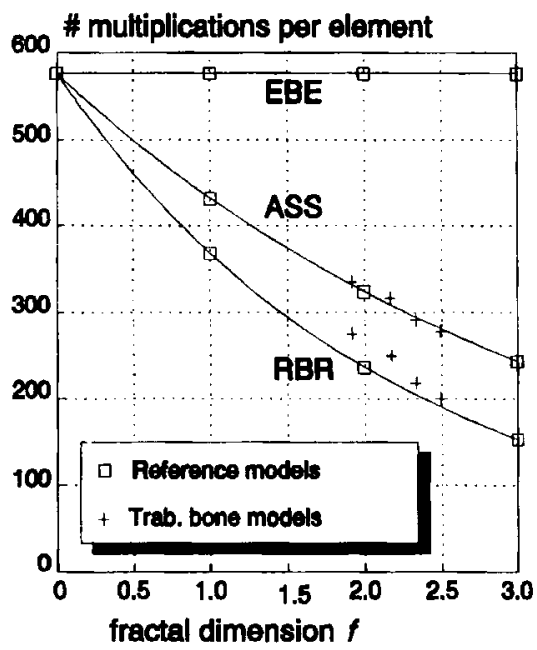

Figure 12. The average number of multiplications per element as a function of the fractal dimension of the FE-model for the EBE, the ASS and for the RBR method

Table VI. Cpu-time per iteration needed to solve the Boussinesq problems

\begin{tabular}{|c|c|c|c|c|c|}
\hline \multirow[b]{2}{*}{$N_{\text {vox }}$} & \multirow[b]{2}{*}{$N_{\text {iter }}$} & \multicolumn{2}{|c|}{ Cray YMP } & \multicolumn{2}{|c|}{ Risc Workstation } \\
\hline & & $\begin{array}{c}\text { EBE } \\
\text { (s) }\end{array}$ & $\begin{array}{c}\mathrm{RBR} \\
\text { (s) }\end{array}$ & $\begin{array}{c}\text { EBE } \\
\text { (s) }\end{array}$ & $\begin{array}{c}\text { RBR } \\
\text { (s) }\end{array}$ \\
\hline $40^{3}$ & 241 & 0.284 & 0.095 & $6 \cdot 10$ & $2 \cdot 43$ \\
\hline $60^{3}$ & 350 & 0.956 & 0.320 & $20 \cdot 48$ & 8.00 \\
\hline $80^{3}$ & 456 & $2 \cdot 263$ & 0.751 & - & - \\
\hline $120^{3}$ & 653 & $7 \cdot 860$ & 2.439 & - & - \\
\hline
\end{tabular}

Table VII. Cpu-time per iteration needed to solve the Trabecular bone problems

\begin{tabular}{|c|c|c|c|c|c|}
\hline \multirow[b]{2}{*}{$N_{\text {vox }}$} & \multirow[b]{2}{*}{$N_{\text {iter }}$} & \multicolumn{2}{|c|}{ Cray YMP } & \multicolumn{2}{|c|}{ Risc Workstation } \\
\hline & & $\begin{array}{c}\text { EBE } \\
\text { (s) }\end{array}$ & $\begin{array}{c}\mathrm{RBR} \\
\text { (s) }\end{array}$ & $\begin{array}{c}\mathrm{EBE} \\
\text { (s) }\end{array}$ & $\begin{array}{c}\text { RBR } \\
\text { (s) }\end{array}$ \\
\hline $40^{3}$ & 1299 & 0.09 & 0.0 & $2 \cdot 16$ & 1.58 \\
\hline $60^{3}$ & 2161 & 0.311 & $0 \cdot 168$ & 6.84 & 4.68 \\
\hline $80^{3}$ & 2673 & 0.779 & 0.347 & $16 \cdot 11$ & 10.04 \\
\hline $120^{3}$ & 4295 & $2 \cdot 351$ & 0.991 & - & - \\
\hline
\end{tabular}

method provide a powerful FE-tool to analyse structures that cannot be analysed in another way. This approach can also be competitive with traditional meshing and solving methods to analyse structures for which voxel data can be obtained in a relative easy way (from, for example, video, Magnetic Resonance Imaging (MRI), or Computed Tomography (CT) images). ${ }^{16}$ 


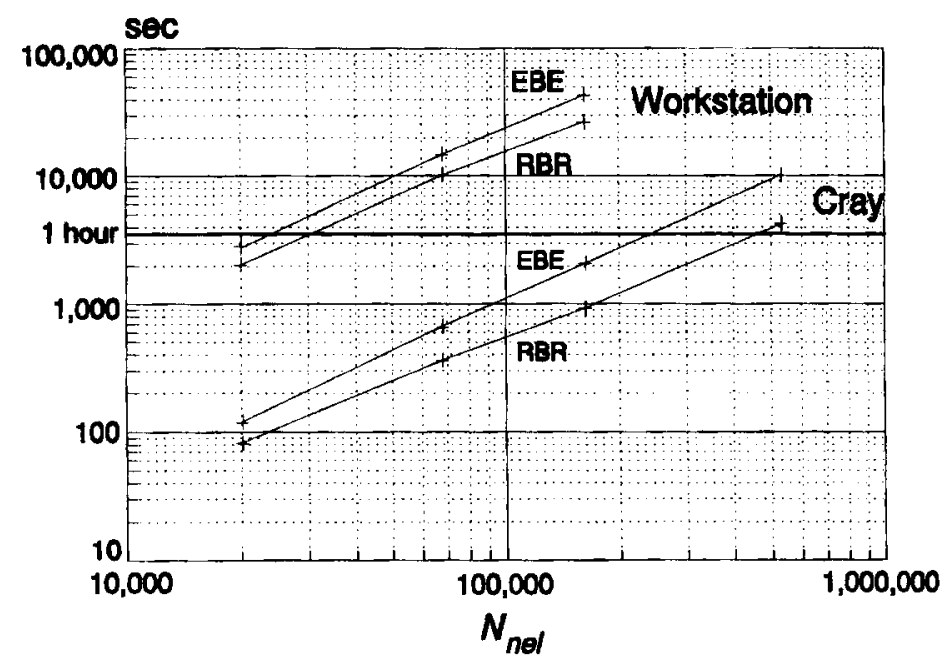

Figure 13. The total cpu-time for a Cray-YMP computer and for a workstation to solve the FE-problems for the trabecular bone models when using the EBE method or the RBR method

The method is essentially a geometric linear FE-method; an incremental element-geometry update results in non-unique elements. However, materially non-linear analysis can be performed with the unique-element EBE strategy. With this method, a different (isotropic) Young's modulus can be specified for each element at costs of storing one additional vector of length $N_{\text {nel }}$ that holds the isotropic Young's modulus for each element. The method then can be used as a subiteration technique for the solution of small deformation materially non-linear problems, whereby the materials remain linear during the solution step and the outside loop is an appropriate non-linear solving algorithm. In another study we have used this approach to simulate the non-linear process of adaptive bone remodelling. ${ }^{17,18}$ Several other researchers have used similar non-linear techniques and FE-models in combination with standard solving techniques to solve optimization and adaptation problems. ${ }^{19-21}$

The present study was focussed on solving linear-elastic FE-models of trabecular bone structure, simply because, as stated in the introduction, there is no other way to solve FEproblems of such irregular structures of this size. The numerical results for the trabecular bone models demonstrate that the EBE and the RBR solution method can be used to solve these problems within reasonable limits of cpu-time and core memory. For the trabecular bone models investigated, the RBR method performs up to 2.37 times faster than the EBE method on the Cray computer, at a cost of using 35 per cent more core memory. An advantage of the EBE method is the fact that a different (isotropic) Young's modulus can be specified for each element. This enables the specification of an element Young's modulus based on the Houndsfield-unit number when using voxel data obtained from (micro-) CT scanning. For the RBR method the effect of different element material properties can be accounted for by separating the contribution of the geometry and the material properties to the matrix entries. The entries can then be constructed by multiplying the geometry factor with the material factor. However, the increased number of multiplications that are needed for this and the fact that no zero entries due to symmetries exist for arbitrary material properties will slow down the performance of this method.

In general, the maximum size of the FE-problems that can be solved with the methods described in this paper is determined by a 'hard limit', i.e. the available core memory, and by a 'soft limit', the available cpu-time. On workstations, the 'hard limit' can be increased by using swap space at cost of using more computer time. For a workstation with 48 Mbyte of memory 
available, the hard limit restricts the size of solvable FE-model to approximately 250000 elements for the EBE or RBR method. In comparison, when the stiffness matrix is fully assembled and stored in compact form, the maximum size would be approximately 30000 elements. Using the EBE and RBR method on a Cray computer with 430 Mbyte of available memory, the size of the FE-models is limited to approximately 2800000 elements.

The cpu time for solving the FE-problems is dependent on the number of elements on the one side and the number of iterations on the other. The cpu time per iteration can be easily estimated from the number of multiplications per increment (equations (5), (9) and (13)) and the performance of the computer. The number of iterations however is largely dependent on the architecture of the FE-model, and on the loading and boundary conditions for the model. For the solid Boussinesq cubes 653 iterative increments are needed to solve the largest model with over 1.7 million elements. For the largest trabecular bone model however, having 69 per cent less elements, 6.6 times more iterations are needed with the same tolerance of convergence. Thus, an estimation of the total cpu time can be obtained only if the number of iterations can be estimated. It must be noted that a judicious use of the convergence criteria can reduce the number of iterations and still provide a sufficiently accurate answer.

It is possible that the numerical results can be improved by the implementation of a more powerful preconditioning method than the diagonal scaling used in the present study. For the EBE solver, element-based preconditioners have been described that can substantially reduce the number of iterations, ${ }^{22.12 .23}$ but the reduction in total cpu-time was much less due to the additional matrix-vector multiplication at the preconditioning stage. The RBR solving method offers a better opportunity for preconditioning since with this method the global stiffness matrix is known 'row-by-row'. Suitable preconditioners would be matrices which can be assembled in the same way as the global stiffness matrix, as used, for example, by the Successive Over Relaxation (SOR) method. ${ }^{8,9}$ The recurrences in the forward-backward substitution schemes with such preconditioners require an element blocking scheme to run in a vectorized way. For this a different data input scheme is needed and therefore such preconditioner methods are not discussed in this paper.

One of the points to be discussed is the inaccuracy introduced by the jagged surfaces in digitized models when compared to the more-or-less smooth surfaces in traditional meshes. To assess the error associated with these surfaces, Hollister et al. ${ }^{5}$ used a two-dimensional model of a void structure that was analysed using both a smooth and a digitized mesh. They found that the errors in the stiffness determination resulting from the digitization are less than 10 per cent for images with $50 \mu \mathrm{m}$ cubic voxels, where typical structures are 100-200 $\mu \mathrm{m}$ in thickness. In an earlier study, ${ }^{24}$ we used a mesh convergence study to estimate the error in the apparent results and in the element stress and strain distribution for realistic three-dimensional trabecular bone structures. In that study it was found that the actual voxel size is not so important as long as the resulting FE-model can describe the trabecular structures (especially the volume fraction) reasonably well. It was found also that for FE-models with an element size of $80 \mu \mathrm{m}$ or smaller, the maximum difference in histograms for the tissue stress and strain distribution was less than 7 per cent when compared to histograms made from a $20 \mu \mathrm{m}$ model. It should be noted that the errors in the actual tissue stress and strain at a specific boundary location may be much higher. On the other hand, the fact that all elements are cubic eliminates the errors found in FE-models that use distorted quadrilateral elements with poor aspect ratios.

\section{ACKNOWLEDGEMENTS}

This study was supported by The Netherlands Foundation for Research (NWO/Medical Sciences), and by the National Computer Facilities Foundation (NCF). 


\section{APPENDIX I}

Determination of node numbers in voxel grid

The voxel data are stored in a three-dimensional array ielgr $(i, j, k)$ which is dimensioned such that it is one voxel larger on each side than the actual data set. For voxels that represent bone $\operatorname{ielgr}(i, j, k)=1$, for voxels that represent void, ielgr $(i, j, k)=0$. A one-dimensional array inogr $(i)$ maps the grid-node position to global node numbers in the FE-model. A two-stage algorithm is used for this. At the first stage bone voxels are searched and the eight grid-nodes that are connected to these elements are marked by setting the corresponding inogr entry to one. At the second stage the grid-nodes thus found are numbered sequentially.

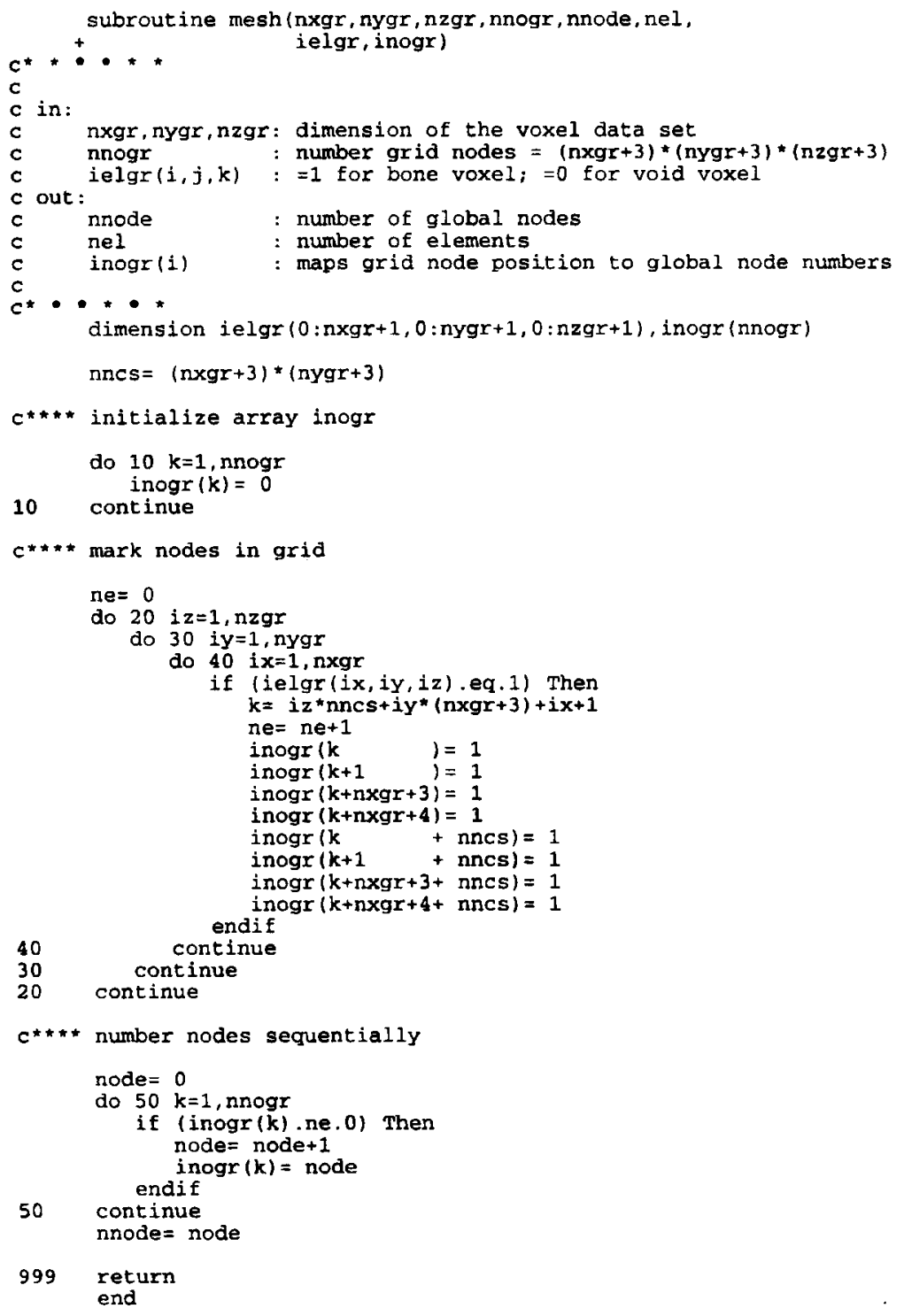




\section{APPENDIX II}

Calculation of case number for each node

The case number of each node is found by checking the element environment for each node in the FE-model. The cases are numbered in accordance with Table II.

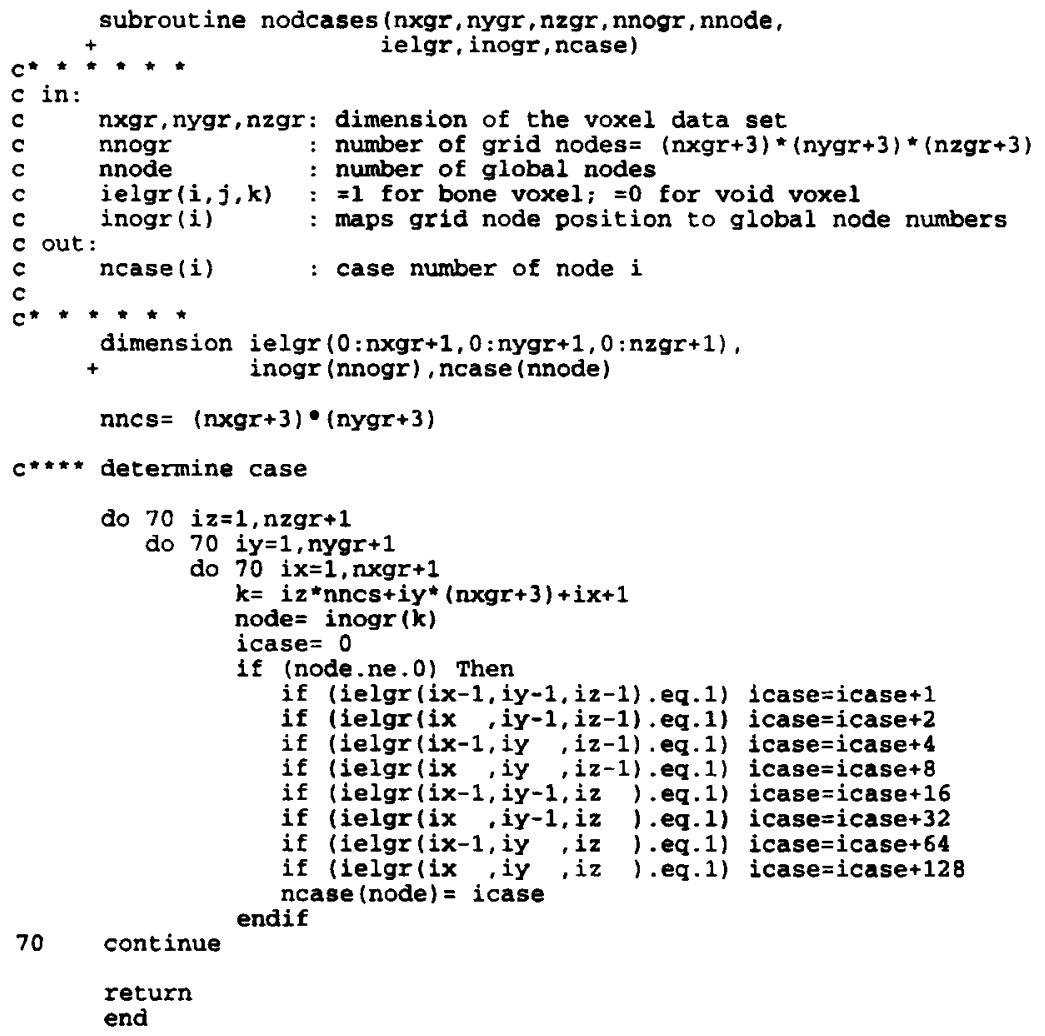




\section{APPENDIX III}

Determination of nodal offset numbers per node, sort and write

Nine offset node numbers, as defined in Figure 8, are calculated for each node in the FE-model. The global node number of the offset nodes is found from array inogr. For offset nodes that are not connected to an element, a value of -1 is found. Output is written to a file.

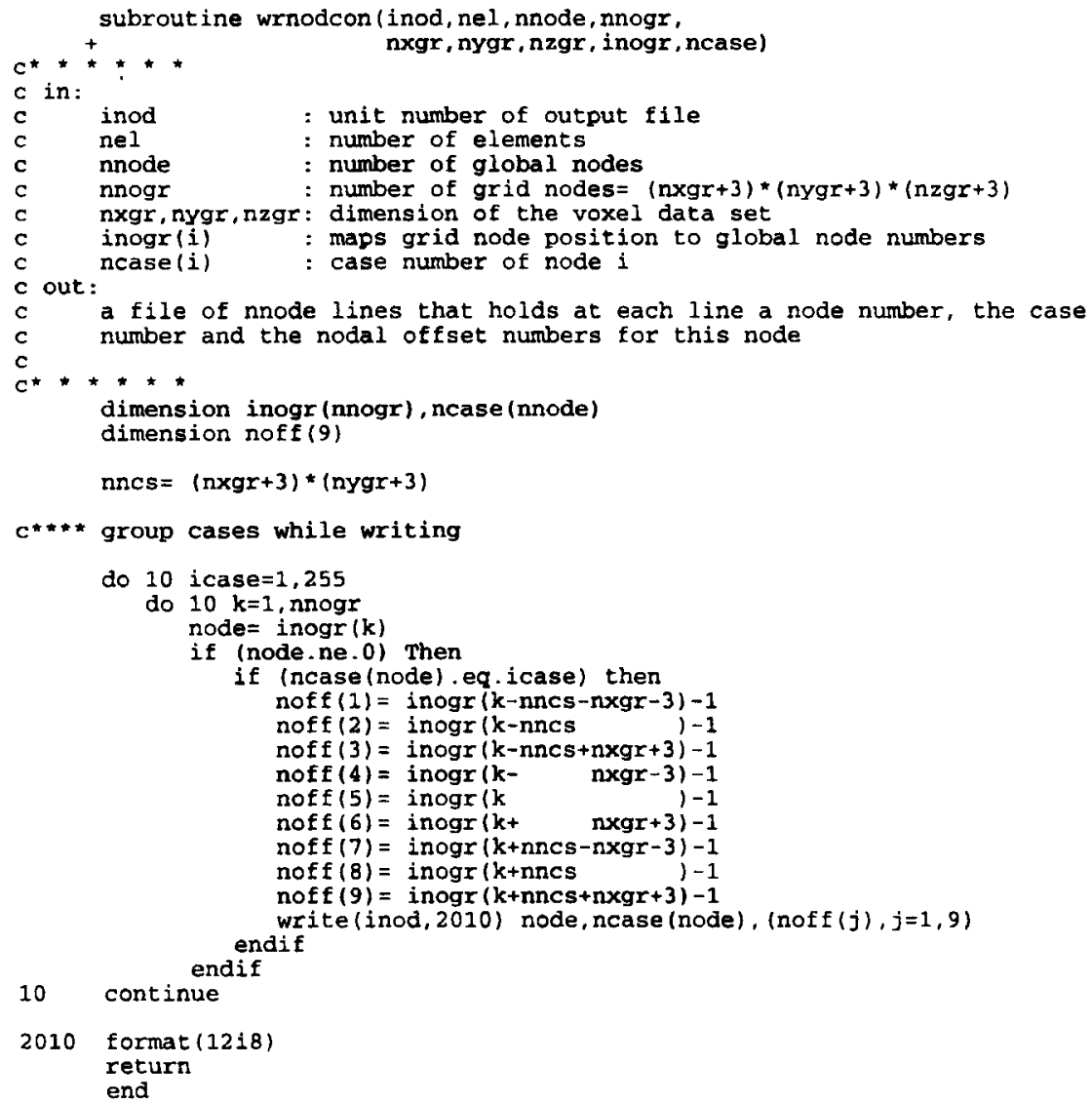




\section{APPENDIX IV}

\section{Read nodal offset and convert to dof offset}

At the start of the solving procedure, the offset nodes are read from a file generated during the preprocessing stage and stored in array noff. The offset node numbers in this array are then converted to the corresponding degree-of-freedom offset numbers needed with the matrix-vector multiplication.

10

20

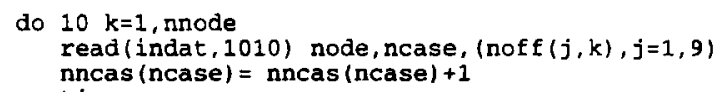

\section{APPENDIX V}

\section{Matrix-vector multiplication routines}

The actual matrix vector multiplication routine is reduced to a case-dependent subroutine call. Since the nodes are sorted per case, all nodes with the same case-number are processed together in the corresponding subroutine.

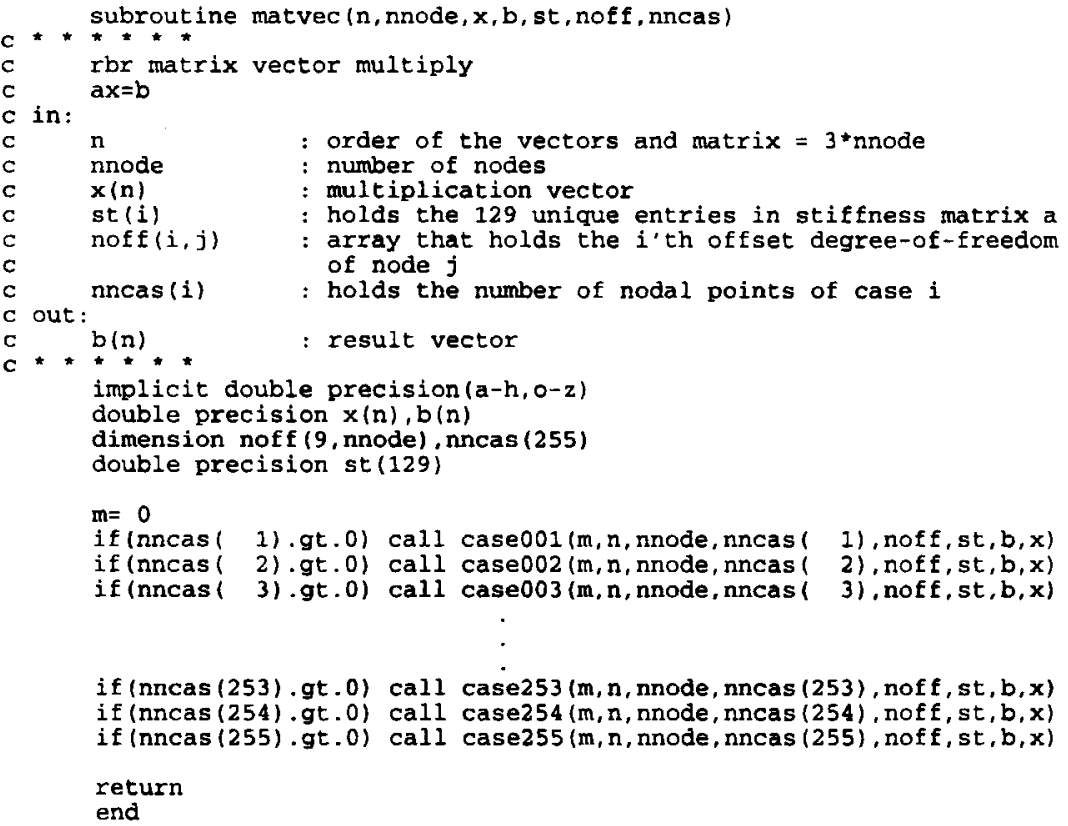


Following is an example of one of these case subroutines. Each routine consists of a loop over the number of nodes for this case. For each node three lines in the stiffness matrix associated with this node are assembled and multiplied with vector $x$. The entries in the stiffness matrix are hard coded' in each subroutine by the indices of array st, their column position in the stiffness matrix is found from array noff. The number and value of the row entries as they appear in the rows of the stiffness matrix are different for each case subroutine.

The loop starting at label 10 can be fully vectorized. Data dependencies with respect to array $b$ cannot occur since each node has a unique offset degree-of-freedom number stored in noff. The compiler directive for the Cray Fortran compiler, prior to this loops, is needed to inform the compiler about this, thus taking care that the loop is vectorized.

Note that the entries in noff can be negative up to -5 (in case a value of -1 was assigned to an offset node, which is converted to an offset degree-of-freedom of -5 as shown in Appendix IV). This implies that vector $x$ must be dimensioned as $x(-5: n)$ in physical memory, with $x(-5: 0)$ filled with zeros. Passed as a dummy argument to subroutines, $x$ can be passed with a 6 position offset and dimensioned with length $n$.

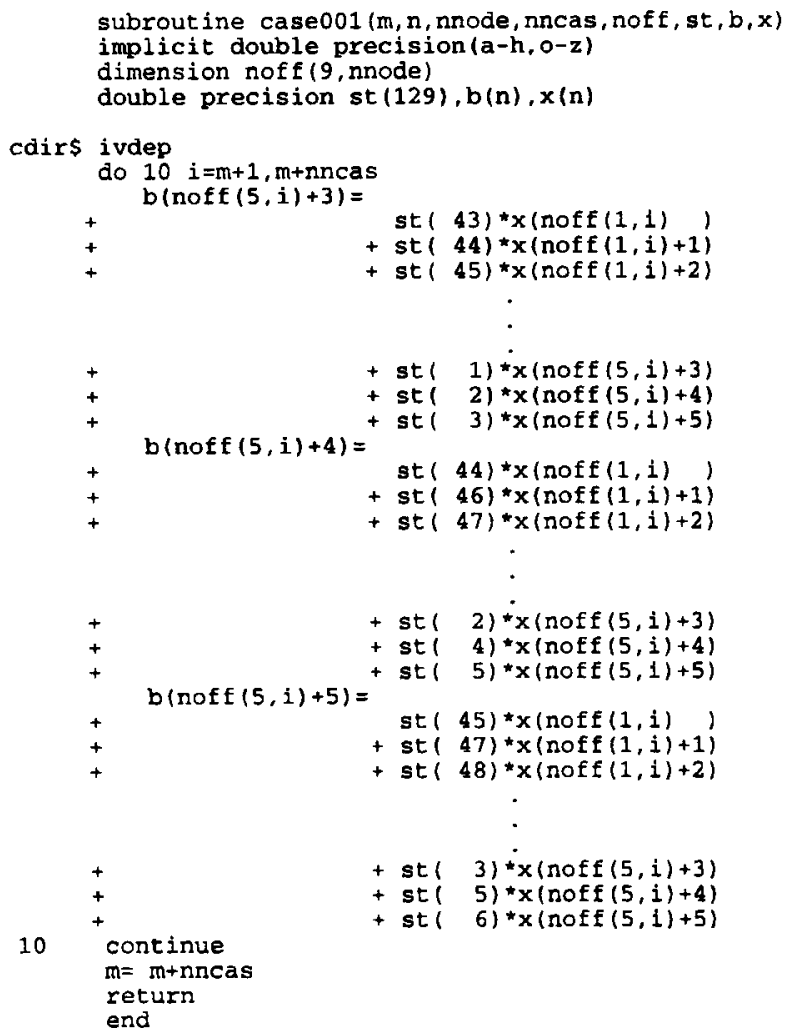

\section{REFERENCES}

1. L. A. Feldkamp, S. A. Goldstein, A. M. Parfitt, G. Jesion and M. Kleerekoper, 'The direct examination of three dimensional bone architecture in vitro by computed tomography', J. Bone Min. Res., 4, 3-11 (1989).

2. A. Odgaard, K. Andersen, F. Melsen and H. J. G. Gundersen, 'A direct method for fast three-dimensional serial reconstruction', J.Microscopy, 159, 335-342 (1990). 
3. A. A. Edidin, J. M. Dawson, M. Zhu and S. Chinchalkar, 'Direct estimation of the modulus of cancellous bone using a variable-stiffness FE model', in Transactions of the 39th Annual Meeting of the Orthopaedic Research Society, Chicago, IL, ORS, 1993. p. 589.

4. D. P. Fyhrie, M. S. Hamid, R. F. Kuo and S. M. Lang, 'Direct three-dimensional finite element analysis of human vertebral cancellous bone', in Transactions of the 38th Annual Meeting of the Orthopaedic Research Society, Chicago, IL, ORS, 1992, p. 551.

5. S. J. Hollister, J. M. Brennan and N. Kikuchi, Recent Advantages in Computer Methods in Biomechanics \& biomedical Engineering, Books \& Journals Int. LTD, Swansea, UK, 1992.

6. S. J. Hollister and N. Kikuchi, 'Direct analysis of trabecular bone stiffness and tissue level mechanics using an element-by-element homogenization method', in Transactions of the 38th Annual Meeting of the Orthopaedic Research Society, Chicago, IL, ORS, 1992, p. 559.

7. T. P. Harrigan, M. Jasty, R. W. Mann and W. H. Harris, 'Limitations of the continuum assumption in cancellous bone', J. Biomech., 21, 269-275 (1988).

8. O. Axelsson and V. A. Barker, Finite Element Solution of Boundary Value Problems, Theory and Computation, Academic Press, Orlando, FL, 1984.

9. G. Strang, Introduction to Applied Mathematics, Wellesley-Cambridge Press, Wellesley, MA, 1986.

10. R. T. Chapman and D. L. Cox, 'A unique element storage implementation of the vectorized element by element preconditioned conjugate gradient algorithm', in I. d. Parsons and B. Nour-Omid (eds.), Iterative Equation Solvers for Structural Mechanics Problems, edited by ASME, New York, 1991, pp. 57-65.

11. I. Fried, 'A gradient computational procedure for the solution of large problems arising from the finite element discretization method', Int. j. numer. methods eng., 2, 477-494 (1994).

12. J. R. Hughes, R. M. Ferencz and J. O. Hallquist, 'Large-scale vectorized implicit calculations in solid mechanics on a cray X-MP/48 utilizing EBE preconditioned conjugate gradients', Comput. Methods Appl. Mech. Eng., 61, 215-248 (1987).

13. R. M. Ferencz, 'Element-by-element preconditioning techniques for large-scale, vectorized finite element analysis in nonlinear solid and structural mechanics', Dissertation, Division of Applied Mechanics, Stanford University and Methods Development Group, Palo Alto, 1989.

14. S. Pissanetzky, Sparse Matrix Technology, Academic Press, London, 1984.

15. M. Seager, 'A slap for the masses', Technical Report, UCRL-100267, Lawrence Livermore Nat. Laboratory, 1988.

16. B. van Rietbergen, H. Weinans, R. Huiskes and B. J. W. Polman, 'A fast solving method for large-scale FE-models generated from computer images, based on a row-by-row matrix-vector multiplication scheme', Proc. $A S M E / C E D$, Vol. 6, pp. 1994 , pp. $47-52$.

17. B. van Rietbergen, M.G. Mullender and R. Huiskes, 'Differentiation to plate-like or strut-like architectures in trabecular bone as a result of mechanical loading', Trans. of the 41. Annual Meeting ORS, 1995, p. 179.

18. B. Van Rietbergen, M. G. Mullender and R. Huiskes, 'A three dimensional for osteocyte-regulated remodeling simulation at the tissue level', in J. Middleton, M. L. Jones and G. N. Pande (eds.), Computer Methods in Biomechanics \& Biomedical Engineering, Gordon and Breach Publishers, 1996, pp 73-83.

19. M. P. Bendsoe, A. R. Diaz, R. Lipton and J. E. Taylor, 'Optimal design of material properties and material distribution for multiple loading conditions', Int. j. numer methods. eng., 38, $1149-1170$ (1995).

20. T. P. Harrigan, J. J. Hamilton, 'Finite element simulation of adaptive bone remodelling: a stability criterion and a time stepping method', Int. j. numer. methods eng., 36, 837-854 (1993).

21. H. Weinans, R. Huiskes and H. J. Grootenboer, 'The behavior of adaptive bone-remodeling simulation models', J. Biomech., 25, 1425-1441 (1992).

22. A. L. G. A. Coutinho, J. L. D. Alves and N. F. F. Ebecken, 'Conjugate gradient solution of finite element equations on the IBM 3090 vector computer utilizing polynomial preconditionings', Comput. Methods. Appl. Mech. Eng., 84, $129-145(1990)$.

23. J. M. Winget, 'Solution algorithms for nonlinear transient heat conduction analysis employing element-by-element iterative strategies', Comput. Methods. Appl. Mech. Eng., 52, 711-815 (1985).

24. B. Van Rietbergen, H. Weinans, R. Huiskes and A. Odgaard, 'A new method to determine trabecular bone elastic properties and loading using micro-mechanical finite-element models', J. Biomech., 28, 69-81 (1994) 